\title{
High current and high power superconducting rectifiers
}

\author{
H.H.J. ten Kate, P.B. Bunk, R.B. Britton and L.J.M. van de Klundert
}

In 1979 a five year project was started to develop a fluxpump for very large currents $(10-100 \mathrm{kA})$ with an energy efficiency of at least $95 \%$ and maximum power under these conditions. A fluxpump is a cryogenic power supply for inductive loads. Because the device works in the cryogenic environment of a superconducting load (a magnet mostly), the heat leak and ohmic dissipation losses in case of high current feed throughs and leads from room temperature to the cryogenic space are avoided for the greater part. It depends on the circumstances and the future capacities of the fluxpump whether it can energize an inductive load completely or can only work as a loss make up device in case of demountable leads. Several reasons: safety, protection and costs for example, have recently caused large superconducting magnets to be designed for very large currents $(5-100 \mathrm{kA})$ and relatively small inductance.

One has to find out whether a fluxpump could be as an alternative to the enormous room temperature rectifiers which are now being planned to deliver such currents. In the case of a fluxpump, the input power leads only carry low currents for controlling and running the fluxpump.

In a review ${ }^{1}$ of the various types of fluxpumps, design criteria have been developed in order to improve the operation of superconducting rectifiers. This review contains a nearly complete list of references dealing with this subject.

As yet research only concerns half wave and full wave rectifịers (Fig. 1a, b). At present the largest current obtained with a full wave rectifier fluxpump is $8 \mathrm{kA} .^{2}$ The diodes normally used at room temperature are replaced now by controlled switches. The requirement of high efficiency forces the rectifier to work in the inductive commutation mode. This means that the secondary current is inductively transferred from one loop to the other avoiding any switching losses. Furthermore, extra loss is avoided by keeping all circuit currents constant during the switching on and off times.

Fig. 1c shows the waveforms of the primary current, both switch control currents and the ideal load current, in the case of a full wave and a halfwave rectifier when loading a purely inductive load. If both switches are closed, the commutation step $\delta I$, which is proportional to the already

R.B.B. is at PO Box 204, Converse Texas 78109, USA. The other authors are at Twente University of Technology, Department of Applied Physics, PO Box 217, 7500 AE ENSCHEDE. The Netherlands. Paper received 7 July 1980. pumped current $I_{\mathrm{L}}$, induces in the secondary circuit that current which results in zero current in the switch to be opened. For $\delta I=0$ the pump operates in the so called resistive commutation mode.

The weakest parts in the superconducting rectifiers are the necessary fast repeating superconducting switches which have to carry the full load current in the on state and which must have enough resistance in the off state to limit leakage currents and ohmic dissipation.

Research mainly concerns the development of these switches. Further topics are ac losses in transformers and switches, high current joints between various types of superconductors as well as protection and control systems for the whole device.

In the next three sections experimental results obtained with three rectifiers are presented.

\section{A thermally switched $1 \mathrm{kA}$ halfwave rectifier fluxpump}

The thermally switched halfwave rectifier now being discussed is shown in Fig. 2.

The primary of the transformer is a solenoid: $5 \mathrm{~cm}$ long, $5 \mathrm{~cm}$ diameter, 3298 turns IMI FM25 NbTi/CuNi conductor, 8 layers, inductance $262 \mathrm{mH}$, central field $65 \mathrm{mT} \mathrm{A}^{-1}$.

The transformer is driven with a $20 \mathrm{~A} / 100 \mathrm{~V}$ amplifier controlled by a signal generator which produces the waveforms shown in Fig. $1 \mathrm{c}$. The secondary coil of the transformer consists of a single turn $(0.1 \mu \mathrm{H})$.

The total secondary circuit is constructed from one type of monolitic conductor (IMI; $3.6 \times 1.8 \mathrm{~mm}^{2} ; 2263 \mathrm{NbTi}$, $35 \mu \mathrm{m}$ fil.; $\mathrm{Cu} / \mathrm{sc}=1.77 / 1$, twist $43.4 \mathrm{~mm} ; I_{\mathrm{c}}=3.4 \mathrm{kA}$ at $5 \mathrm{~T}$ ).

The three joints in the circuit are made by soldering $(97 \mathrm{~Pb}$ $3 \mathrm{Ag}$ ) the copper matrixes with an overlap of $3 \mathrm{~cm}^{2}$. A great advantage of using one type of conductor is that less joints have to be made (at least 2) and that difficult joints between different types of conductors are unnecessary. The total resistance of the three joints together was measured via the decay time of the secondary circuit $(L / R=4.4 \mathrm{~h})$ and is $2 \times 10^{-10} \Omega$.

For demonstration purposes there is no need for a big load inductance. The only condition is that the load coil inductance $(2.6 \mu \mathrm{H})$ is much larger than the inductance of the shortcircuited secondary of the transformer $(0.45 \mu \mathrm{H})$. The 


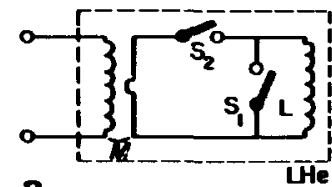

a

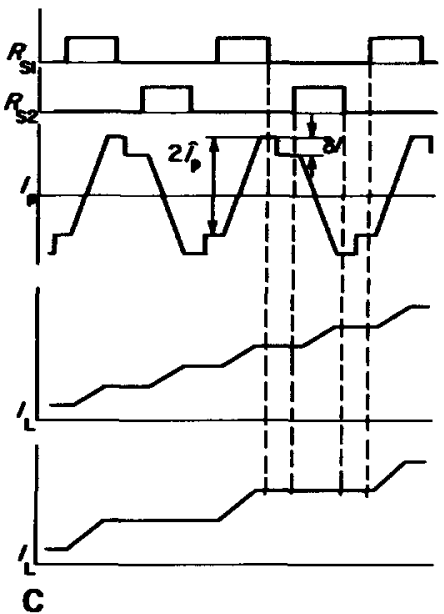

b

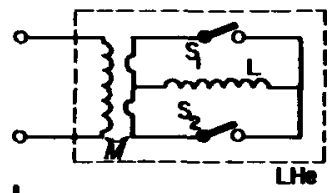

Fig. 1 Schemes of a superconducting a - half wave and $b-$ full wave rectifier. c - Waveforms of both switch resistances $R_{\mathbf{s} 1}$ and $R_{\mathrm{s} 2}$ with primary current $I_{\mathrm{p}}$ and load current $I_{L}$ in the case of a full and half wave rectifier

load coil consists of 8.5 turns on a $5 \mathrm{~cm}$ diameter, $5 \mathrm{~cm}$ long coilform. The central field is $0.15 \mathrm{~T} \mathrm{kA}^{-1}$.

The thermal switches (Fig. 3 ) are made by etching away the copper matrix of the conductor over $2.5 \mathrm{~cm}$. A nichrome heater wire is wrapped bifilarly around the bundle of filaments. A cylindrical insulation jacket of STYCAST 2850 FT reduces the necessary heater power to $60 \mathrm{~mW}(45 \mathrm{~mA}$ into $30 \Omega$ ). The resistance of the switch is $7.5 \mathrm{~m} \Omega$ at $300 \mathrm{~K}$ and $3 \mathrm{~m} \Omega$ at $T=T_{\mathrm{c}}$.

The current in the secondary coil of the transformer is known from a Rogowski pick-up toroid plus an integrator which actually measures the self-field of the current. The current in the load coil is measured with a Hall probe (Siemens RHY 18).

The Hall voltage is fed back to the waveform generator to regulate the communication step $\delta I$ in the primary current $I_{\mathrm{p}}$ (Fig. 1c).

The current amplification in the shortcircuited situation (both switches closed) is 260 and 30 when the load is connected (switch $S_{1}$ open). The theoretical maximum load current $\left(\phi / l_{\mathrm{sec}}=2 \hat{I_{\mathrm{p}} M} / l_{\mathrm{sec}}\right)$ for this geometry is $500 \hat{I}_{\mathrm{p}} \mathrm{A}$, apart from conductor limitations. Above $1100 \mathrm{~A}$ the conductor in the switches quenches due to the instability of the conductor there. This result was to be expected because a degradation of the critical current by a factor of seven is quite normal in the absence of the copper matrix, especially in the low field region $0.5-1.5 \mathrm{~T}$. To overcome this phenomenon one has to find a good compromise between decreased stability and sufficient resistance of the conductor when the switch is open. Otherwise more parallel switches can be used or one has to give up the concept of using only one type of conductor in the secondary circuit.

This fluxpump operated at frequencies from $0.1 \mathrm{up}$ to $10 \mathrm{~Hz}$. Above $1 \mathrm{~Hz}$ the $1100 \mathrm{~A}$ is not reached because the switches do not cool down completely to $4.2 \mathrm{~K}$ so that the critical current is lowered further. Fig. 4 shows the loading process for the cases of the resistive $(\delta I=0)$ and two inductive commutation modes.

If the commutation step $(\delta I)$ is added to the constant amplitude of the primary current (Fig. $4 \mathrm{~b}$ ) a linear curve is obtained.

$$
I_{\mathrm{L}}=\frac{2 \hat{\mathrm{I}}_{\mathrm{p}} M}{L} \mathrm{ft} \mathrm{A}
$$

If, however, the commutation step $(\delta I)$ is substracted from the amplitude of the primary current (Fig. 4c) and in the resistive cummutation mode $(\delta I=0)$ (Fig. 4a), the same exponential shape of the load curve is found.

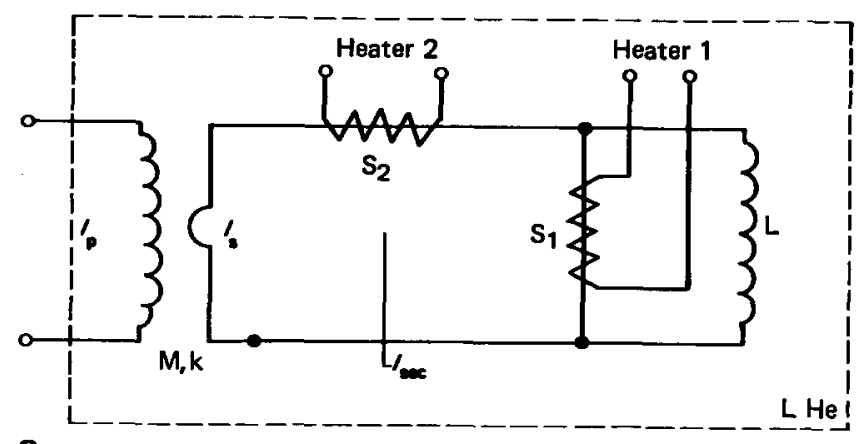

a

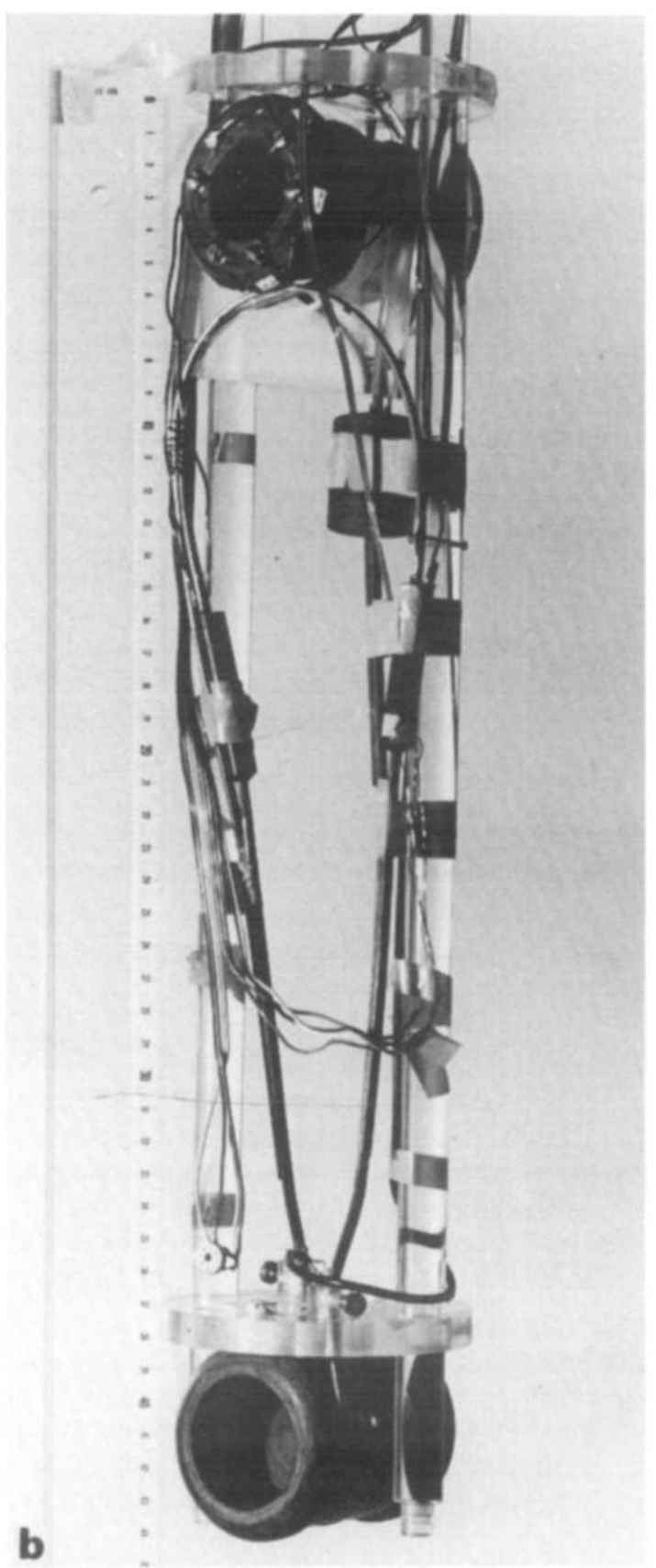

Fig. 2 a - Scheme and circuit parameters of thermally switched half wave rectifier, $I_{\text {sec }}=0.45 \mu \mathrm{H}, L=2.6 \mu \mathrm{H}, k=0.72, I_{\mathrm{p}}=262 \mathrm{mH}$, $M=116 \mu \mathrm{H}, I_{\mathrm{s}}=0.1 \mu \mathrm{H}, \mathrm{b}-$ View of the half wave rectifier 


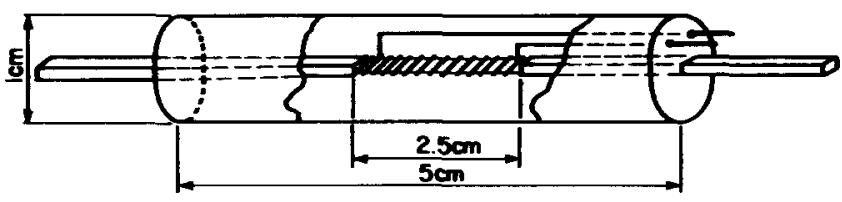

Fig. 3 Thermal switch of a monolithic high current conductor
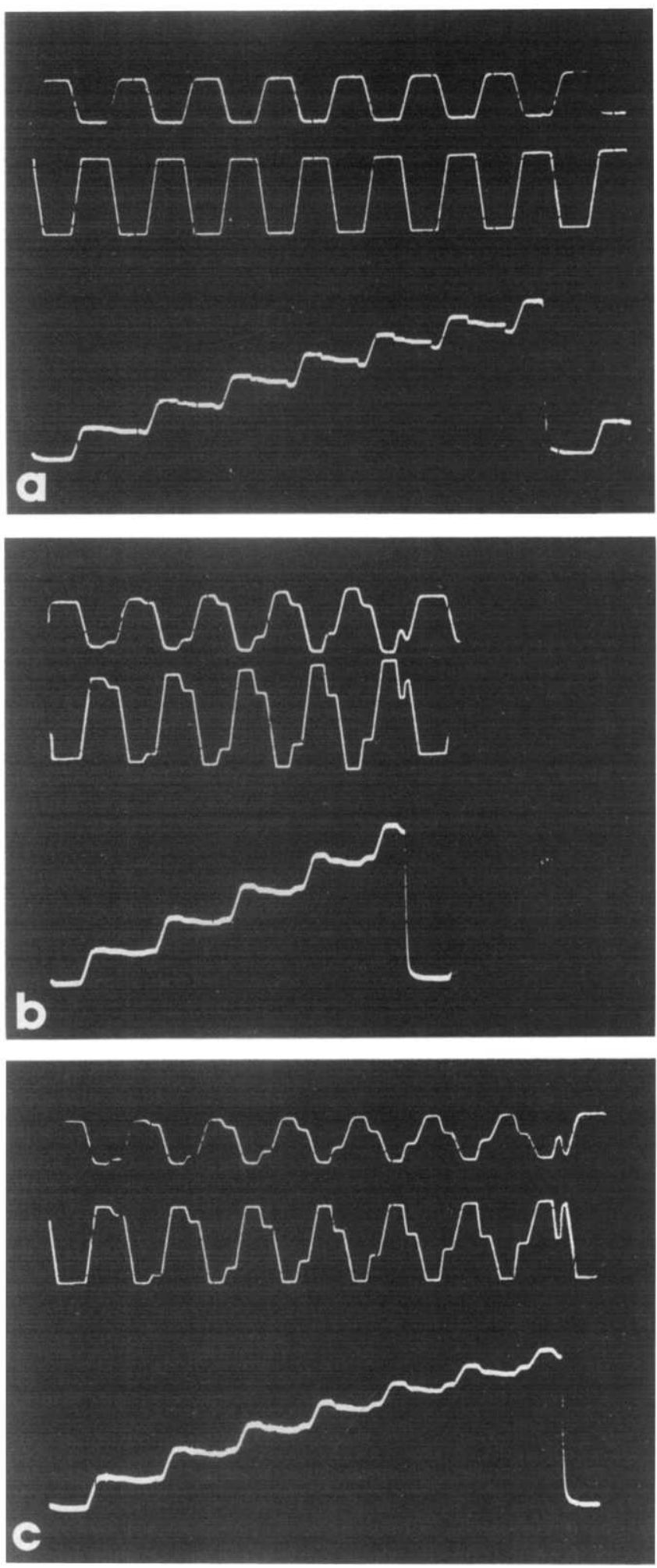

Fig. 4 Loadcurves of the half wave rectifier flux pump for a resistive commutation mode $(\delta /=0) ; b$ - inductive commutation mode with the commutationstep $\delta /$ added to the constant amplitude of the primary current; $\mathrm{c}$ - inductive commutation mode with the commutation step $\delta /$ subtracted from the amplitude of the primary current. On each photograph one sees, from top to bottom, the primary current, the generator waveform and the loadcurrent

$$
I_{\mathrm{L}}=\frac{2 \hat{I}_{\mathrm{p}} M}{l_{\mathrm{sec}}}\left[1-\exp \left(-\frac{f l_{\mathrm{sec}} t}{L}\right)\right] .
$$

At $1100 \mathrm{~A}$ the secondary circuit quenches and the stored energy (1.5 J) is dissipated.

When the amplitude of the primary current $\left(\hat{I}_{\mathrm{p}}\right)$ is $3.7 \mathrm{~A}$ at $0.2 \mathrm{~Hz}$, the maximum current of $1100 \mathrm{~A}$ is reached within $35 \mathrm{~s}$ with an average power of $40 \mathrm{~mW}$ (Fig. 4a, c).

The decrease of the load current at the time that $S_{2}$ is opened is clearly seen in Fig. 4a. This current loss is proportional to the load current itself and is due to the loss of the stored energy in the secondary circuit except the load coil.

This energy is dissipated when $S_{1}$ opens. These decreases of the load current are absent in the inductive commutation mode (Fig. 4b, c). Unwanted magnetic couplings between transformer and the shortcircuited load coil causes steps down and up in the load current curve.

\section{A 1 kA magnetically switched full wave rectifier}

The experimental set up and electrical scheme of the magnetically switched full wave rectifier is shown in Fig. 5. The primary of the transformer for this pump is the same as the one in the previous section.

The complete secondary circuit is made of a niobium foil conductor (KBI INC; $106 \times 0.025 \mathrm{~mm}^{2} ; I_{\mathrm{c}} \approx 1.5 \mathrm{kA}$ at $0.2 \mathrm{~T} ; T_{\mathrm{c}} \approx 9 \mathrm{~K}$ ). The twice doubled foil ( $27 \mathrm{~mm}$ wide) is used to make a load coil and the rest of the secondary circuit except the transformer. The two secondary turns of the transformer consist of three times doubled foil (14 mm wide).

The six joints in the secondary circuit are spotwelded. One spot for every $50 \mathrm{~A}$ is sufficient. A decay time $(L / R)$ of the secondary circuit of more than 125 days determines the combined resistance of the six joints to less than $10^{-12} \Omega$. The load coil has 15 turns on a $56 \mathrm{~mm}$ diameter, $53 \mathrm{~mm}$ long coilform, an inductance of $11 \mu \mathrm{H}$ and a central field of $0.24 \mathrm{~T} \mathrm{kA}^{-1}$. The current amplification is 900 in the shortcircuited situation when both switches are closed and 8.5 when the load is connected (one switch open).

The secondary currents are measured in the same way as described in the previous section

The magnetic switches consist of a noninductive shaped superconductor which is transfered to the normal state in an overcritical magnetic field $\left(B_{\mathrm{c} 2} \approx 0.37 \mathrm{~T}\right)$.

One meter niobium foil is bifilarly folded in a sandwich geometry (Fig. 6) which gives a normal resistance of 2.5 $\mathrm{m} S 2$ at $4.2 \mathrm{~K}$. The foil is cut into four parallel strips of $2.65 \mathrm{~cm}$ width which are put on top of each other.

Then the foil is zigzagged to form the bifilar geometry. An oxidized aluminium strip lies between every two niobium layers. Because the oxide on the aluminium surface was too vulnerable, extra plastic insulation was required (Fig. 7).

The rectangular switch element is pressed between the two halves of the coil form. Simple solenoids make a central field of $50 \mathrm{mT} \mathrm{A}^{-1}$ parallel to the conductor surface. The lengths of the switch coils and the niobium part of the switch element are 5 and $2.65 \mathrm{~cm}$ respectively. The magnetic field at the edges of the niobium is about $60 \%$ of the central field. 


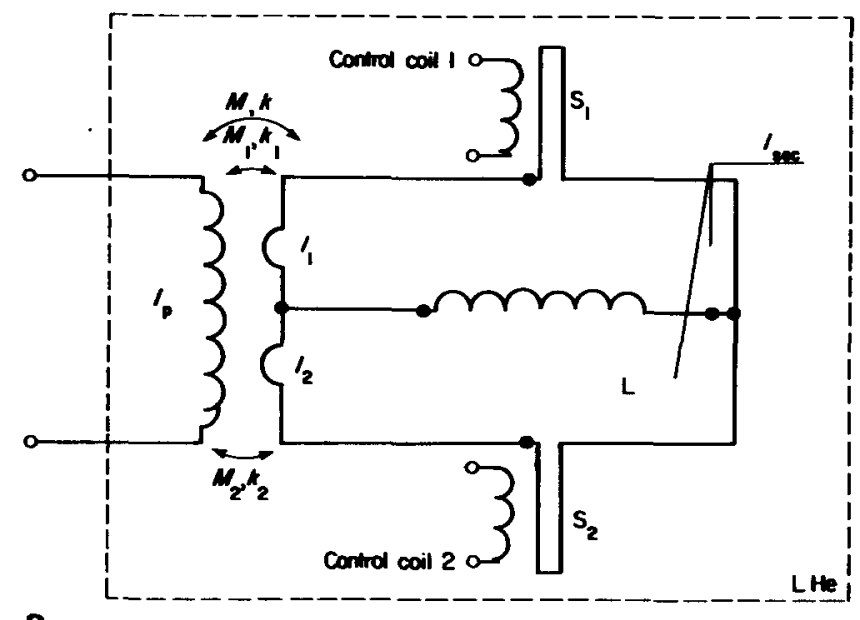

a

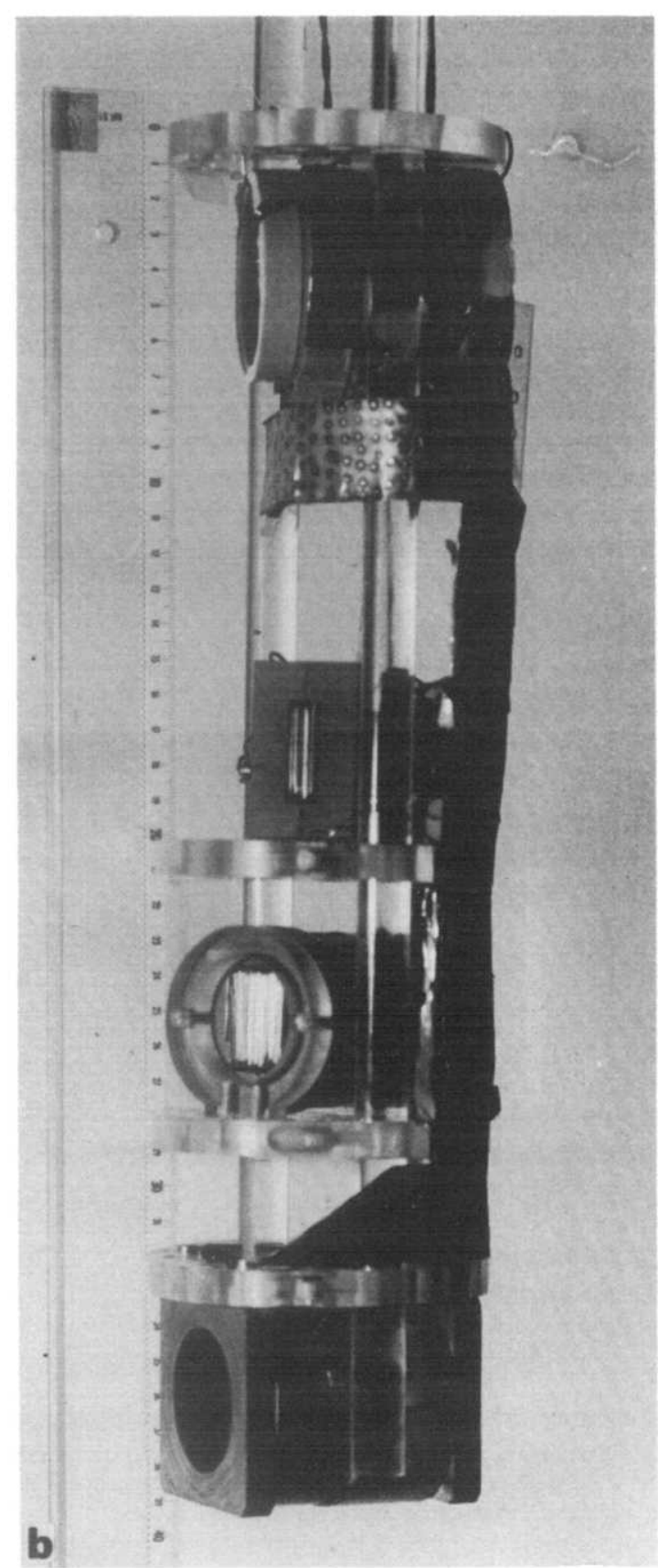

Fig. 5 a - Scheme and circuit parameters of the magnetically switched full wave rectifier $I_{\mathrm{p}}=262 \mathrm{mH}, M=M_{1}+M_{2}=85+80$ $=165 \mu \mathrm{H}, l_{1}=I_{2}=0.07 \mu \mathrm{H}, I_{\text {sec }}=0.18 \mu \mathrm{H}, L=11 \mu \mathrm{H}, k_{1}=k_{2}$ $=0.6, k=0.36 \mathrm{~b}-\mathrm{View}$ of the full wave rectifier

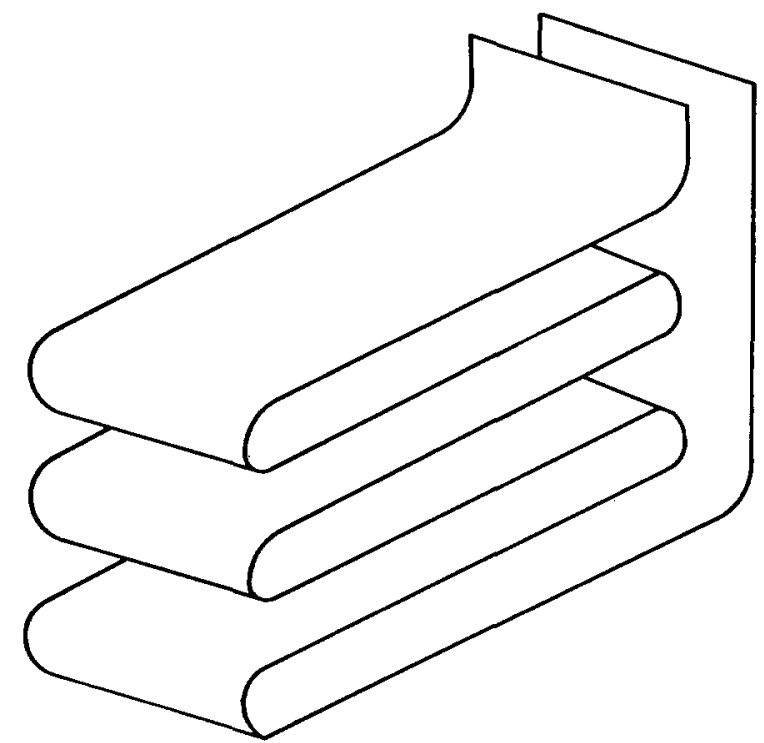

Fig. 6 Non inductive arrangement of the switch element

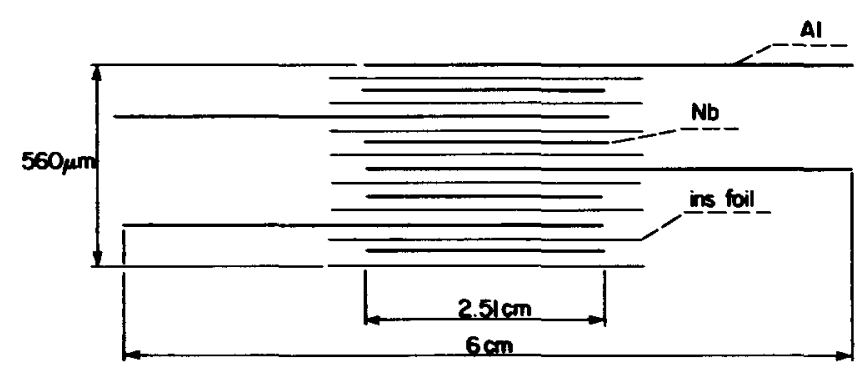

Fig. 7 Cross section of the switch conductor

The aluminium strips lie in the direction of the coil axis and protrude into the helium to form cooling ribs. At $T=T_{\mathrm{c}} \approx 9 \mathrm{~K}$ this switch geometry can conduct a stationary heating power of $15 \mathrm{~W}$.

Although a perpendicular field on the superconductor is preferred, this is not possible while still retaining the aluminium cooling fins. Besides, the perpendicular field would cause undesirable eddy current losses in the niobium and aluminium.

The theoretical maximum current in this geometry $\left(I_{\max }=\phi_{0} / l_{\mathrm{sec}}=2 \hat{I}_{\mathrm{p}} M / l_{\mathrm{sec}}\right)$ is $1800 \hat{I}_{\mathrm{p}} \mathrm{A}$.

In the frequency range below $1 \mathrm{~Hz}$ a stable current of $1000 \mathrm{~A}$ can be pumped.

Usually the circuit quenches during the resistive or inductive commutation part of a cycle (large $\mathrm{d} J / \mathrm{d} t$ ). Sometimes it happens during the increase of the load current.

Considering the critical current density of the niobium, the magnetic field in the load coil and the self field of the current between the close parallel conductors, a larger load current cannot be expected. Niobium is unsuited as a high current conductor.

Remarkable is the perfect shape of the load curve in both resistive and inductive commutation mode (Fig. 8). The inductive commutation with constant primary amplitude is shown here. If the load curve is linear then:

$$
I_{\mathrm{L}}=\frac{4 f \hat{I}_{\mathrm{p}} M}{L} t
$$

Also there is a complete absence of unwanted coupling and current loss for a magnified case (not shown in the figure). 


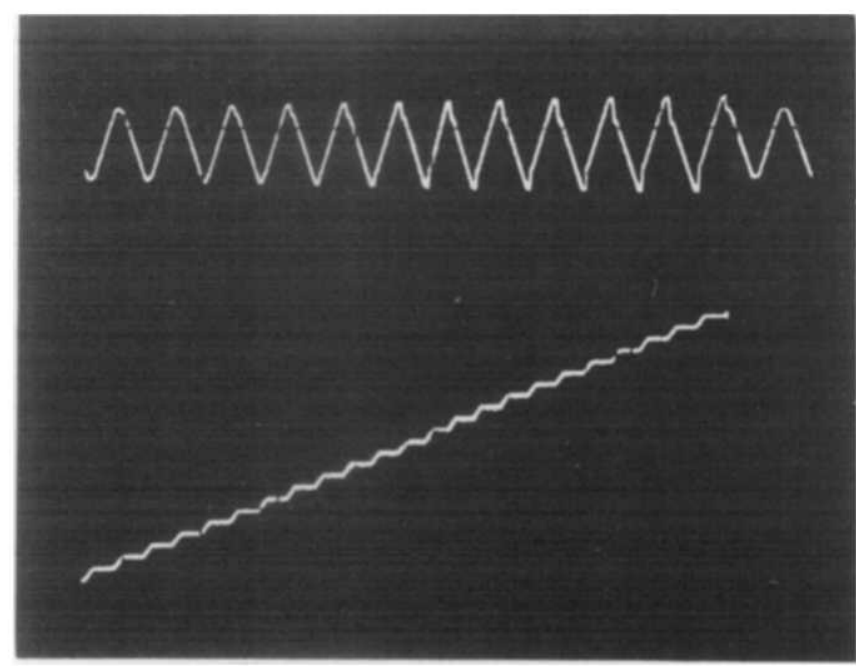

Fig. 8 Primary current and loadcurve of the full wave rectifier fluxpump. The commutation step $\delta /$ is added to the amplitude of the primary current resulting in a linear loadcurve. At $1 \mathrm{kA}$ the circuit quenches

\section{A magnetically switched $50 \mathrm{~Hz}$ full wave rectifier}

The frequency of operation in magnetically switched fluxpumps is limited by ac losses and the inductances of the switch field coils. When decreased, efficiency is acceptable for certain applications the use of $50 \mathrm{~Hz}$ mains and directly derived waveforms can be attractive. ${ }^{3}$ The power of the rectifier can be increased considerably by a factor of 2500 compared to the previous low frequency, large current rectifiers.

A transformer and both switches are connected as shown in Fig. 9. The rectifier is tested with a pure ohmic load outside the cryostat. The brooks coil transformer is wound on a glassfibre epoxy cylinder (53 $\mathrm{mm}$ diameter). From the inside moving outwards, the packet of windings consists of eight primary layers, $2 \times 4$ secondary layers and again

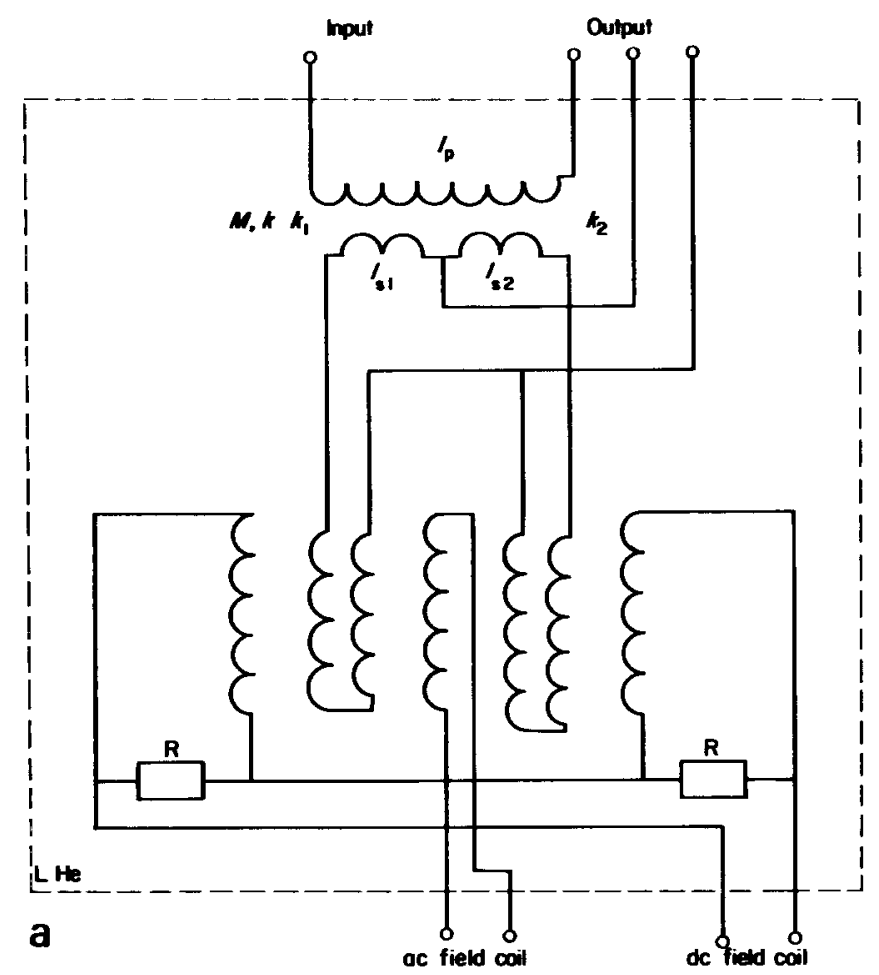

seven primary layers. Between all conductor layers there is an extra layer of thin parallel copper wires to conduct the heat outwards. The primary is wound with Supercon $\mathrm{Nb} 25 \mathrm{Zr}, \mathrm{Cu} / \mathrm{sc}=1.67 / 1,203 \mu \mathrm{m}$ wire; the secondary with Supercon $\mathrm{Nb} 48 \mathrm{Ti}, \mathrm{Cu} / \mathrm{sc}=0.67$, single core, $483 \mu \mathrm{m}$ conductor. During a separate test the transformer could produce $45 \mathrm{~A} \mathrm{rms}, 520 \mathrm{~W}$ at $37 \mathrm{~Hz}$ and $20 \mathrm{~A} \mathrm{rms}, 100 \mathrm{~W}$ at $50 \mathrm{~Hz}$. The transformer losses were about $1 \%$ at $50 \mathrm{~Hz}$.

Both switches have been combined in one geometry. Wound on a $59 \mathrm{~mm}$ diameter glassfibre epoxy cylinder there are successively the first do field coil, one switch element, an ac field coil, the other switch element and the second dc field coil. The coils are spaced with thin nylon wire to allow helium flow. Every two layers a layer of thin copper wires allows for more helium penetration between the layers and extra cooling. Both nylon and copper wires lie in the direction of the coil axis with mutual distance of about $1 \mathrm{~mm}$.

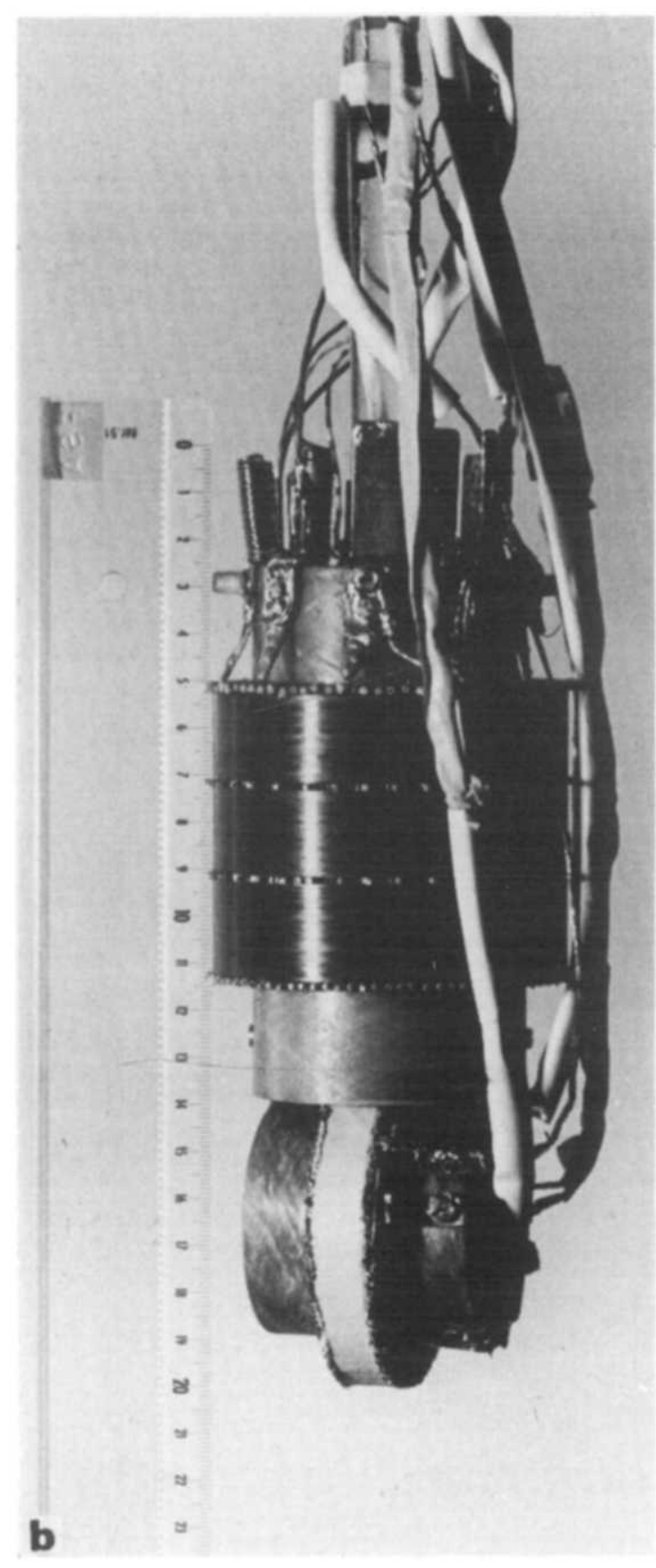

Fig. 9 a - Scheme and circuit parameters of the $50 \mathrm{~Hz}$ full wave rectifier $I_{\mathrm{p}}=34 \mathrm{mH}, I_{\mathrm{s} 1}=I_{\mathrm{s} 2}=1.4 \mathrm{mH}, M=9.0 \mathrm{mH}, k_{1}=k_{2}=0.92$, $k=0.94, I_{\text {switch element }}=32 \mu \mathrm{H}, L_{\text {dc coils }}=18.2 \mathrm{mH}, L_{\text {ac coils }}=113 \mathrm{mH}, R=0.2 \mathrm{~m} \Omega ; \mathrm{b}-$ View of the $50 \mathrm{~Hz}$ rectitier 


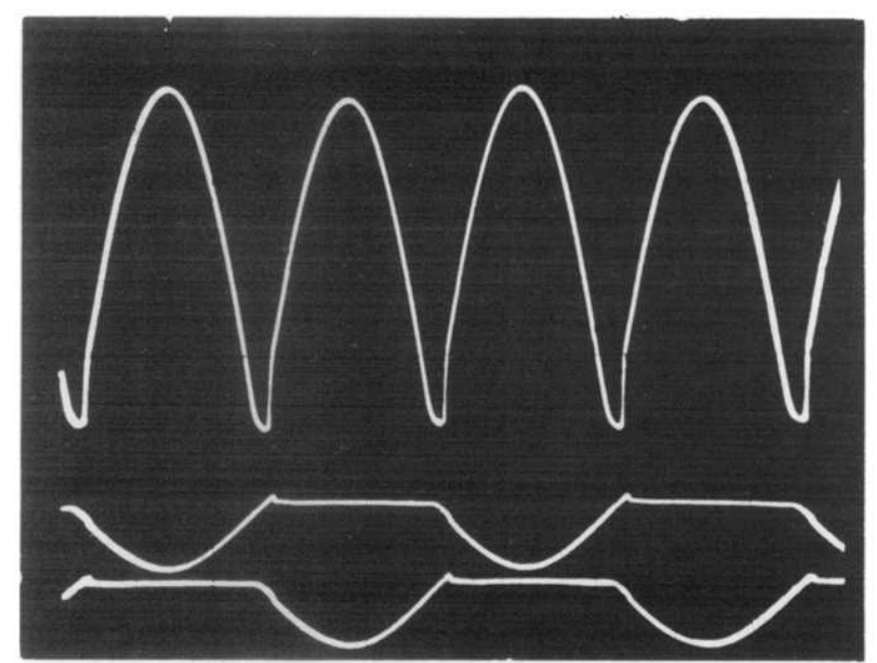

Fig. 10 Output current and both gate currents of the high frequency full wave rectifier with a purely ohmic load

The two switch elements are bifilarly wound of $70 \mathrm{~m}$ Supercon $\mathrm{Nb} 1 \mathrm{Zr}, \mathrm{Cu} 30 \mathrm{Ni}, 18$ filament, twisted, $305 \mu \mathrm{m}$ conductor which has a second critical field of about $0.85 \mathrm{~T}$. When fully normal the gate resistance is $32 \Omega$ at $4.2 \mathrm{~K}$.

The $\mathrm{dc}$ field coils are wound from IMI NbTi/Cu, $330 \mu \mathrm{m}$ wire. The ac field coil is made of Supercon $\mathrm{Nb} 25 \mathrm{Zr}, \mathrm{Cu} / \mathrm{sc}$ $=1.67 / 1,203 \mu \mathrm{m}$ conductor. Across both dc coils there are small resistors $(0.2 \mathrm{~m} \Omega)$ to shortcircuit induced ac currents. The dc and ac coils produce a magnetic field of $20 \mathrm{mT} / \mathrm{A}$ and $14 \mathrm{mT} / \mathrm{A}$ respectively.

Dependent on the direction of the current in the ac coil the magnetic fields are added and subtracted at the switch elements successively. The dc coils produce a subcritical field at both switch elements.

The field of the ac coil alternately drives the gates normal. There is a time interval in which both gates are superconducting. This depends on the value of the bias field. During this time interval, the current is transferred from one gate to the other.

Whether or not the current transfer will be complete depends upon several factors such as switching on and off times. Generally a mixture of inductive and resistive commutation takes place. The current through the ac switch coil has to be nearly in phase with the secondary current in the transformer Otherwise the switch conductor goes normal because the field dependent critical current is exceeded.

Several tests have been performed with sinusoidal input currents. At 15, 25 and $50 \mathrm{~Hz}$ the rectifier supplies a stable power of 120,100 and $60 \mathrm{~W}$ rms respectively into a $80 \mathrm{~m} \Omega$ resistor outside the cryostat. At 15 and $25 \mathrm{~Hz}$ the total cryogenic losses of the whole device are about 5 and $10 \%$ whereby the transformer loss is less than $1 \%$. Fig. 10 shows the rectified output current and both gate currents at $25 \mathrm{~Hz}$. The total magnetic field in the switches is swept between 0.60 and $0.88 \mathrm{~T}$. The decrease of the obtainable power with increasing frequency is caused by increasing ac losses limiting the current through the ac field coil. Moreover the whole device operates in the field range of $0.5-1.5 \mathrm{~T}$ where conductor stabilization is problematic.

The authors are indepted to $\mathrm{Mr}$ M. Caspari for design and construction of the waveform generator and to Mr H.A. Steffens for his valuable assistance during the construction of the fluxpumps.

\section{References}

1 van de Klundert, L.J.M., ten Kate, H.H.J. On fully superconducting rectifiers and fluxpumps. A review. Cryogenics 21 (1981) 195

2 Spiel, D.E., Boom, R.W. Superconductive energy storage with a fluxpump, Int Inst of Refrigeration Low Temp and Electric Power Conf London (1969) Pergamon Press, NY (1970) 433

3 Britton, R.B., Abbatielly, F.J., Robins, K.E. Fluxpumps and ac superconducting components, 4th Int Conf on Magnet Technology, Brookhaven BNL 17225 (1972) 703 